\title{
Network structure and metropolitan mobility
}

\author{
Pavithra Parthasarathi \\ CDM Smith $^{\text {a }}$
}

\begin{abstract}
This research aims to develop quantitative measures that capture various aspects of the underlying network structure, using aggregate level travel data from fifty metropolitan areas across the US. The influence of these measures on system performance is then tested using statistical regression models. The results corroborate that the quantitative measures of network structure affect the system performance. The results from this analysis can be used to develop network design guidelines that can be used to address current transportation problems.
\end{abstract}

\section{Introduction}

Networks shape cities and how people use them. Yet, the explicit consideration of the deep structure of the transportation network is largely missing from the models that explain how cities work and how travelers behave within them. Streets form the primary structural element of any city. The differences in modern cities such as New York or Los Angeles trace back to the transportation system in place during critical phases of growth for each city (Marshall 2005). The spatial structure of modern cities arise from their transportation and communication infrastructure (Anas et al. 1998). An in-depth analysis of urban design and travel needs to explicitly consider the transportation network in terms of the structure, the actual layout of streets and routes.

This research investigates how cities work by examining a cross-section of US cities. The use of cross-sections to explain performance is a feature of much influential research in the field. Famously, Newman and Kenworthy (1989) argued that the variation in transportation use between the US and other countries mainly reflects differences in land-use patterns and transportation systems. This argument was countered by Gordon and Richardson (1989) and Gomez-Ibanez (1991) who criticized the theoretical and methodological foundations used in the study and the proposed policy recommendations. This debate has continued ever since (van de Coevering and Schwanen 2006).

Bento et al. (2003) analyzed the influence of urban form and public transit supply on travel demand using national level travel survey data from 114 urbanized areas in the US. Giuliano and Narayan (2003) provide an international perspective by comparing travel diary data from the US and Great Britain. The results indicated that the differences in mobility patterns could be explained by the differences in both urban form and household income. Levine et al. (2009) related transportation accessibility outcomes to urban form using data from twenty four metropolitan areas in the US. Other metropolitan level comparisons include the role of monocentric and polycentric urban structures in influencing travel and its associated effects (ex. environmental costs, social costs etc) (Schwanen et al. 2001; Veneri 2010).

A review of the literature on urban form and travel shows a wide variety of modeling methodologies and research approaches (Crane 2000; Ewing and Cervero 2010; Krizek 2003; Stead 2001). A key aspect missing from this body of work on urban form is the explicit consideration of the underlying transportation network structure. Some would argue that if you have travel times between places, the

a parthasarathip@cdmsmith.com

Copyright 2014 Pavithra Parthasarathi.

Licensed under the Creative Commons Attribution - NonCommercial License 3.0. 
network structure is unimportant. That is, while network structure may explain journey time, since it is those journey times that travelers care about, network structure is already accounted for. Recent research suggests otherwise. While travel time is of course important, it is insufficient as people systematically misperceive time and value time in different conditions differently (Parthasarathi et al. 2013). Network structure enables us to explain some of the misperceptions and different valuations.

This research aims to further the research interest in understanding travel behavior while explicitly accounting for the underlying street network structure, using aggregate level travel data from fifty metropolitan areas across the US. The argument presented here is that while the metropolitan transportation network need not be the only indicator of travel in a region, an understanding of the relationship between network architecture and travel is essential for the design of sustainable and efficient cities. The question of how travel behavior varies systematically with network structure is particularly important as network architecture is perhaps the slowest changing urban system. For that reason it is the most important to get right, as the design of the network persists for centuries and is difficult to adjust, much less optimize.

This paper aims to quantify the structure of street networks of fifty metropolitan areas and to understand their influence on two aspects of transportation (street) network performance, namely congestion and highway system use. In addition to their usefulness as measures of system performance, they also have particular importance for environmental analyses, which often use vehicle distance traveled as an input.

The rest of the paper is organized as follows. The next section briefly reviews relevant literature. This is followed by the section on modeling methodology, data sources, and the estimation of measures of roadway network structure. The model formulation and key hypothesis are then identified. The statistical analyses conducted and the results follow in the next section. The paper concludes with key findings from the study and future extensions to the current research.

\section{Network structure}

Network structure comprises scale, topology, hierarchy, and morphology, including the layout (arrangement and connectivity) of the network and characteristics of individual elements (Kissling 1969; Xie and Levinson 2007). Borchert (1961) conducted one of the earliest studies on this topic, using network measures to understand metropolitan settlement patterns. In parallel, Garrison and Marble (1961) analyzed the relationship between the structure of transportation networks and characteristics of the area in which the networks are located. Kansky (1963) used graph theory to develop a wide range of network measures to quantify the spatial structure of transportation networks (railways and roadways). The interest in understanding network structure using graph theory shifted in the 1970s due to advances in computers and subsequent focus on developing complicated transportation models (Derrible and Kennedy 2010).

Recent advances in GIS capabilities and related spatial analysis software has revived the interest in understanding the topological properties of complex networks. Yang et al. (2009) developed a method to identify and classify the spatial (grid-like) patterns in road networks with complicated junctions. Other advances include the use of fractal geometry and complex network theory to understand the patterns, structure and evolution of transportation networks (Jiang and Claramunt 2004; Kim et al. 2003; Lu and Tang 2004; Yan and Wang 2009).

Barabasi and Bonabeau (2003) focused on scale-free networks in an attempt to understand the underlying principle governing extremely complex systems such as the world wide web. Salingaros $(1998,2000)$ used structural principles from other fields such as biology, computer science and economics to understand the fundamental processes behind urban design. His research focused not just on identifying the principle elements but also on understanding the connectivity and the complexity 
and coherence essential for successful urban design. Jenelius (2009) used Swedish road network data to relate the long-term vulnerability of the network to its underlying structure. Gu et al. (2012) developed indicators of network structure to understand the overall characteristics and the spatial structure of the Chinese railway network.

Xie and Levinson (2007) developed complementary measures of network structure to better understand and quantify the structural attributes of complicated road networks. The measures of network structure were initially developed using idealized test networks and later applied to the Swiss road networks to trace the changes in network characteristics over time (Erath et al. 2009).

These advances in network analysis have allowed researchers to focus on the relationship between network structure and travel. Patuelli et al. (2010) conducted an analysis of German commuting trends from both a spatial and network perspective to understand the hierarchies among 439 German districts. Zhang et al. (2012) analyzed the association between road network structure and nonmotorist safety using data from census tracts in Alameda County, California.

Levinson and El-Geneidy (2009) used a measure of network circuity to better understand the relationship between residential location choice relative to work using data from the Twin Cities metropolitan region. Derrible and Kennedy $(2009,2010)$ characterized the network structure of 33 metro systems around the world using graph theory based measures. The analysis was extended to study the relationship between the estimated network measures and transit ridership using data on a subsample of 19 subway systems. The results of the regression model show a strong relationship between the network measures and ridership indicating the importance of network design in attracting people to transit systems. In a recent cross-sectional study, Levinson (2012) systematically compared the variation in network structure across the 50 largest metropolitan areas in the US. The analysis showed the variation in network structure with city size confirming that larger cities are physically more inter-connected.

Our current paper aims to extend this interest in network analysis to road networks across the US and relate it the system performance and usage.

The following measures of street network structure are estimated for each of the fifty metropolitan areas:

- Hierarchy

Entropy

Percentage of freeways

- Topology

Treeness

Completeness

Average circuity

- Scale

Street density

\subsection{Entropy}

Road networks are heterogeneous, considering the differentiated functional designs and operational performance of hierarchical roads.

The entropy measure of heterogeneity is given as:

$$
H(X)=-\sum_{i=1}^{m} p_{i} \log _{2}\left(p_{i}\right)
$$


where,

$m=$ Number of subsets in the system $X$,

$p_{i}=$ Proportion of elements in the $i^{\text {th }}$ subset.

Individual links can be grouped into subsets based on different road properties such as functional type, traffic volume, capacity, or level of service. In this paper, entropy is defined by functional class. Functional classification is the process by which highways are classified into groups, based on the type of service they are expected to provide (Federal Highway Administration 2000a). Typical classifications include arterials, collectors, local streets, etc.

Entropy captures the differentiation that exists among road networks. Roadway networks typically have a very high proportion of local streets compared to other functional categories. The differentiation in network structure, as measured by the entropy variable, identifies a variation from this typical condition. Hence a higher entropy measure indicates the presence of higher functional classification links in the network such as arterials compared to local streets.

\subsection{Percentage of freeways}

This measure is also designed to capture the hierarchy in real-world street networks. It is similar to the above entropy measure but focuses specifically on the freeways in the area. The percentage of freeways is estimated as:

$$
\% \mathrm{~F}=\frac{L_{f}}{L} * 100
$$

where,

$L_{f}=$ Freeway kilometers in the area,

$L=$ Total roadway kilometers in the area.

This measure is estimated for each metropolitan area in the dataset.

\subsection{Treeness}

This measure is based on the two basic structures of a planar transportation network: circuit and tree (Haggett and Chorley 1969). A circuit is defined as a closed path, with no less than three links, that begins and ends at the same node. A tree is defined as a set of connected lines that do not form a complete circuit. A regional network distinguished by closed circuits is therefore called a circuit network while a network defined by a tree shaped structure is called a branching network. Refer to Xie and Levinson (2007) for a complete description of this measure.

Open source software ${ }^{1}$ was used to classify each segment in the street network as belonging to a branch network or a circuit network. This code was implemented on the street network of each metropolitan area. The street networks, as mentioned above, were obtained from the US Census Bureau.

The treeness for each street network was then estimated as:

$$
\phi_{\text {tree }}=\frac{L_{t}}{L}
$$

where,

$L_{t}=$ Length $(\mathrm{km})$ of street segments belonging to a branch network in the area,

$L=$ Total roadway kilometers in the area.

\footnotetext{
${ }^{1}$ Developed by Feng Xie, Metropolitan Washington Council of Governments (MWCOG); code can be downloaded from http://nexus.umn.edu/Software/IdentifyingNetworkTopologies.zip
} 
The treeness measures captures the differences in topology and connection patterns that exist among real-world street networks. Treeness can also be considered to be a measure of organization (in)efficiency.

\subsection{Completeness}

This measure, as the name indicates, captures the level of completeness in the network using a link-node approach. This measure is similar to network connectivity measures, proposed earlier by Garrison and Marble (1961) and Kansky (1963). Road networks are typically characterized by links and nodes. Links refer to the street segments while nodes refer to the intersection or junction of two or more links. Considering a road network with $E$ links (edges) and $V$ nodes (vertices), the network is said to be 100 percent complete if each node in the network is directly connected to each of the remaining nodes.

Figure 1 shows the relationship between the maximum number of direct connections in the network and the number of nodes in the network.

The number of links in a real world network is typically less than the maximum number of links and the completeness index used here captures this difference. The completeness $\left(\rho_{e}\right)$ of the street network is defined as:

$$
\rho_{e}=\frac{E}{V^{2}-V}
$$

The street networks obtained from the Census TIGER/line files were cleaned to ensure that the network contained only the relevant links and intersection or junction nodes. Shape nodes included in the TIGER/line files to ensure spatial correctness were removed as they do not represent actual intersection or junctions.

\subsection{Average Circuity}

Network circuity is defined as the ratio of the shortest path network distance to the Euclidean or straight-line distance between an origin and destination (OD) pair. Levinson and El-Geneidy (2009) used a dataset of randomly selected origins and destinations of actual trips to estimate circuity in their analysis of commute patterns and compared that to random OD points. The same methodology is implemented here, finding that circuity of actual home to work trips was lower than random OD points of the same trip length. Here we use circuity of random trips constrained to match actual trip length, which is highly correlated with (but higher than) actual commute circuity.

For each study area in our dataset, two samples were generated. The first sample consists of the origins and the second sample consists of the destinations. Similar to Levinson and El-Geneidy (2009), 200 randomly distributed origins and 1000 randomly distributed destinations were generated using GIS. This provided $200^{*} 1000$ OD pairs for each area resulting in a 200,000 OD matrix. The network distance and the Euclidean distance were calculated for each of 200,000 OD pairs.

A subsample of OD pairs were selected out from the 200,000 random OD matrix in each study area by matching the network distance to the average commute trip length, provided in the $2001 \mathrm{Na}-$ tional Household Travel Survey (NHTS) (Federal Highway Administration 2001). The average circuity for the subsample of OD pairs in each area was then estimated as:

$$
C=\frac{D_{n}}{D_{e}}
$$

where,

$C=$ Average circuity in the metropolitan area, 

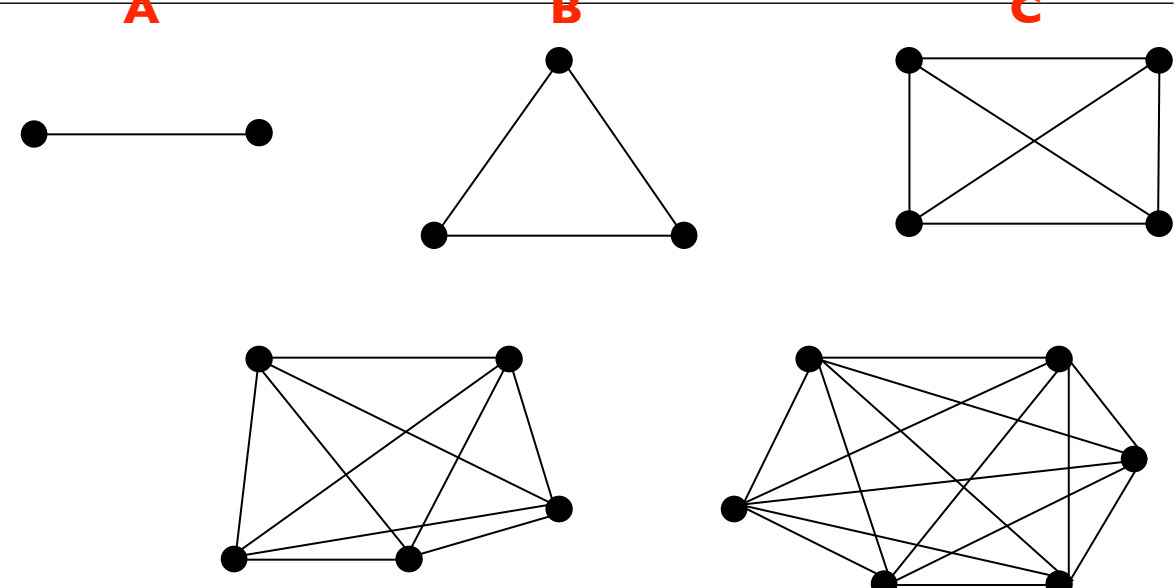

D

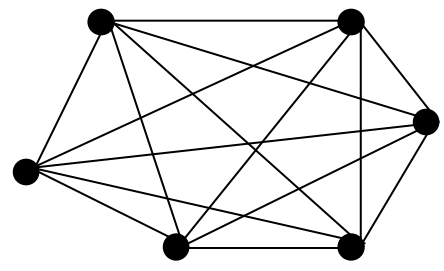

E

\begin{tabular}{|c|c|c|c|c|}
\hline Network & Nodes & $\begin{array}{c}\text { Maximum number } \\
\text { of I-way links }\end{array}$ & $\begin{array}{c}\text { Maximum number } \\
\text { of 2-way Links }\end{array}$ & Completeness \\
\hline A & 2 & 1 & 2 & $100 \%$ \\
\hline B & 3 & 3 & 6 & $100 \%$ \\
\hline C & 4 & 6 & 12 & $100 \%$ \\
\hline D & 5 & 10 & 20 & $100 \%$ \\
\hline E & 6 & 15 & 30 & $100 \%$ \\
\hline
\end{tabular}

Figure 1: Maximum number of links in sample networks

$D_{n}=$ Sum of the network distance $(\mathrm{km})$ between all OD pairs in the subsample,

$D_{e}=$ Sum of the Euclidean distance $(\mathrm{km})$ between all OD pairs in the subsample.

The circuity measure was estimated for 48 of the 50 study areas in our dataset. The circuity could not be estimated for two study areas, namely Richmond, Virginia, and Birmingham, Alabama, due to the unavailability of commute data for these areas in the 2001 NHTS. Please refer to Levinson and El-Geneidy (2009) for a detailed explanation of the methodology.

The circuity measure is designed to capture the inefficiency in the network from the view point of a traveler.

\subsection{Street density}

Street Density $\left(\rho_{l m}\right)$ for each metropolitan area is estimated as:

$$
\rho_{l m}=\frac{L}{A}
$$

where,

$L=$ Roadway kilometers in the area,

$A=$ Size of the area $\left(\mathrm{km}^{2}\right)$

The estimated measure has a unit of $1 / \mathrm{km}$. This measure differs from the completeness measure in that it provides a measure of the size of the actual network in comparison to the size of the urban area. The completeness, on the other hand, does not account for the urban area size and looks at the connectivity of the network using basic principles of graph theory. The street density can be considered to be a measure of the network intensity, while the completeness is a measure of the efficiency in network connectivity. The correlation matrix presented at the end of this section confirms that the two measures are not correlated with each other. 


\subsection{Accessibility}

Accessibility refers to the ease of reaching destinations or activities (Levinson 2012). Hansen (1959) began the examination of accessibility as part of a study to develop a residential land-use model. Horton and Reynolds (1971) provide a geographical perspective in their evaluation of the effects of urban spatial structure and accessibility on individual travel. A review on this topic shows many methods to estimate accessibility in the region (El-Geneidy and Levinson 2006). The cumulative opportunity measure is one such method; it estimates the number of destinations $\left(O_{t}\right)$ that can be reached in a given time threshold.

A similar approach is used here to measure the average number of people that can be reached in 30 minutes by automobile at uniform average metropolitan density. The accessibility measure is estimated as:

$$
O_{t}=\pi *\left[\frac{S_{n} \cdot t}{C}\right]^{2} * \rho_{p m}
$$

where,

$\rho_{p m}=$ Urban area population density $\left(\right.$ person $\left.\cdot \mathrm{km}^{-2}\right)$.

$t=$ time threshold (30 minutes in this analysis)

$S_{n}=$ Average network speed in $\mathrm{km} / \mathrm{h}$

$C=$ Average circuity, as estimated above

The above formulation incorporates both the density aspect of urban areas and the structure of the street network. The accessibility was estimated for 48 of the 50 study areas due to the lack of appropriate circuity measures for two study areas.

A correlation matrix of the above estimated network measures is provided in Table 1 . The lack of high correlation between any of the estimated measures of network structure in Table 1 confirms that the variables are measuring different aspects of network structure.

Table 1: Correlation of estimated measures

\begin{tabular}{|l|l|l|l|l|l|l|l|}
\hline & $\% F$ & $H(X)$ & $\phi_{\text {tree }}$ & $\rho_{e}$ & $C$ & $\rho_{l m}$ & $O_{30}$ \\
\hline $\begin{array}{l}\text { Percentage of freeways, } \\
\% F\end{array}$ & 1 & & & & & & \\
\hline Entropy, $H(X)$ & $\mathbf{0 . 3 4 9 2}$ & 1 & & & & & \\
\hline Treeness, $\phi_{\text {tree }}$ & 0.2082 & $\mathbf{0 . 3 3 6 6}$ & 1 & & & & \\
\hline Completeness, $\rho_{e}$ & 0.176 & 0.1928 & -0.134 & 1 & & & \\
\hline Average circuity, $C$ & 0.1581 & -0.2055 & $\mathbf{0 . 5 0 4 2}$ & -0.0368 & 1 & & \\
\hline Street density, $\rho_{l m}$ & -0.2761 & $\mathbf{- 0 . 4 1 9 3}$ & -0.18 & -0.0186 & 0.2603 & 1 & \\
\hline Accessibility, $O_{30}$ & -0.2056 & -0.1995 & $\mathbf{- 0 . 3 8 5 4}$ & 0.1145 & -0.1401 & $\mathbf{0 . 4 8 1 2}$ & 1 \\
\hline bold - Indicates significant at 95\% confidence level & & & & \\
\hline
\end{tabular}

\section{Data}

This research aims to understand the systematic variation in transportation system performance with measures of network structure, using data from the top fifty metropolitan areas across the US. The metropolitan areas were selected based on the year 2000 population data obtained from the US Census Bureau.

The first step in this analysis is to obtain the relevant network and non-network data for the metropolitan areas considered in the study. The primary data for this empirical analysis comes from the following sources: 


\subsection{Street networks}

The street networks for the fifty metropolitan areas, used in this analysis, were extracted from the Census TIGER/line files. The Topologically Integrated Geographic Encoding and Referencing (TIGER) files, developed and maintained by the US Census Bureau, provide information on various features such as roads, railroads, rivers, as wells as legal and statistical geographic areas (U.S. Census Bureau 2008). The extracted networks for the metropolitan areas were cleaned to include just the road features based on the Feature Class Codes (FCC) for the line segments provided in the Census TIGER/Line files.

\subsection{Travel data}

The travel data is from the Texas Transportation Institute's Urban Mobility Report and provides information on the long-term congestion trends and congestion comparisons for 90 urban areas across the US (Schrank and Lomax 2009). The data typically includes information on the:

- System usage - Vehicle miles traveled (VMT), annual passenger miles, unlinked transit passenger trips

- System supply - Highway lane miles by functional category

- Congestion measures - Annual hours of delay on the system, annual cost of congestion, congestion indexes like the Travel Time Index (TTI) and the Roadway Congestion Index (RCI).

The data on highway system usage was also supplemented by data from the Federal Highway Administration (FHWA)'s Highway Performance Monitoring System (HPMS), a national-level continuing database that summarizes important statistics on the condition and performance of the highway system (Federal Highway Administration 2000b). The travel data for the year 2000 was extracted for the purpose of this analysis.

\subsection{Socio-demographic data}

The socio-demographic data was obtained for the year 2000 from the US Census Bureau for the fifty metropolitan areas considered in the analysis. The socio-demographic variables are used as control variables in our analysis.

\subsection{Delay, vehicle travel per capita, and network structure}

Before getting into the details of the two models (congestion, system usage), a simple analysis of the relation between select network measures and select system performance measures is presented here. Figure 2 shows how delay (darker points indicate more delay) and vehicle travel per capita (wider points indicate more Vehicle Kilometers Traveled (VKT) per capita) vary with entropy and circuity. Mean values for each quintile are reported in Table 2.

As the network becomes more varied (which generally means more hierarchical, including more links at the top of the hierarchy (a greater share of freeway links)), delay decreases, while for networks with low entropy, there is generally more delay. As the network becomes more circuitous (on the right), delay increases, while less circuitous networks have less delay. The least delay occurs in the upper left corner, the most delay in the lower right. Metropolitan areas with the least delay have the most VKT per capita, and the shortest Journey to Work Time, indicating that in uncongested areas people travel much more by automobile for non-work trips than people in the most delayed quintile.

The analysis presented here shows a relationship between transportation system performance and select network measures. The actual pattern of influence is identified using the two regression models presented in the following sections. 


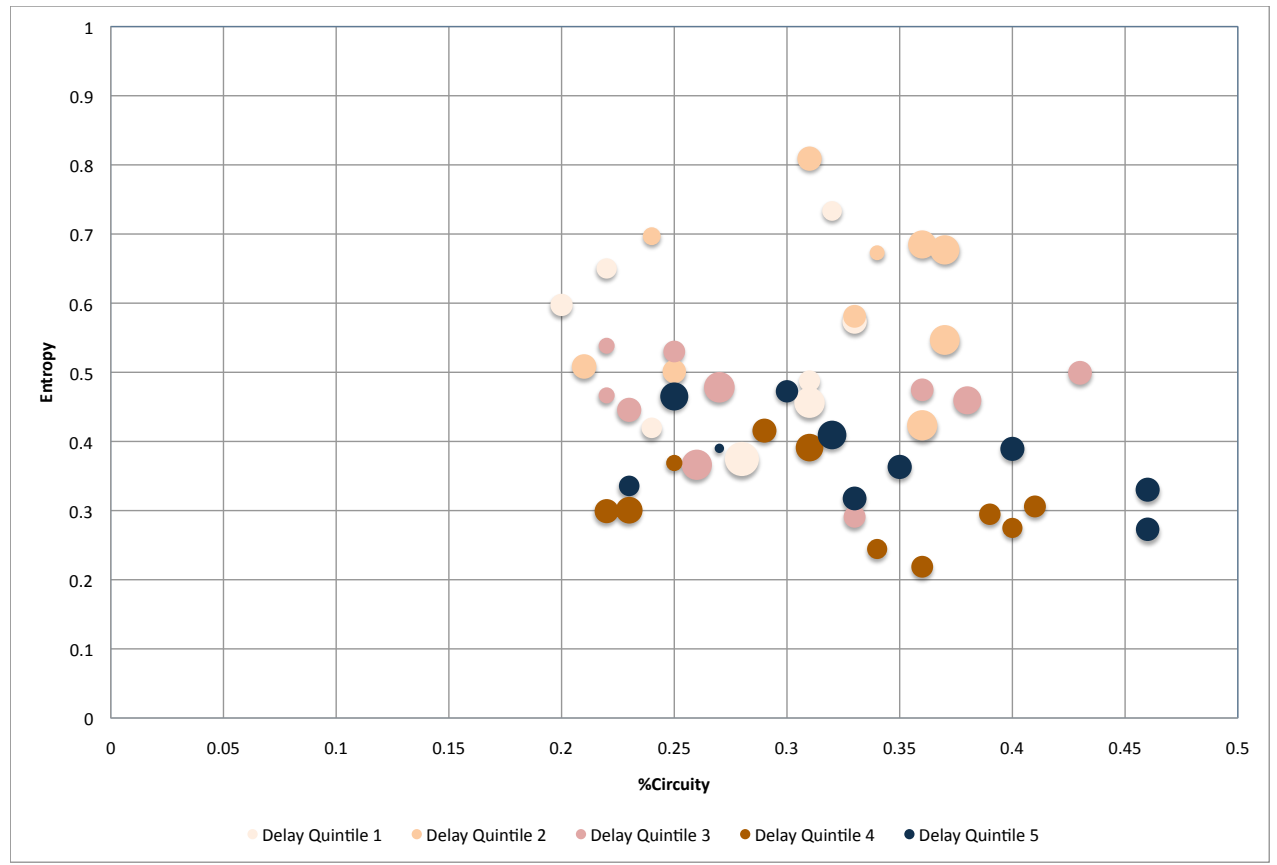

Figure 2: Variation in system performance as a function of select network measures (circuity, entropy)

Note:

Delay Quintile 1 represent least delay;

Delay Quintile 5 represents most delay;

Darker points indicate more delay;

Wider points indicate more Vehicle Kilometers Traveled (VKT) per capita.

Table 2: Mean values tabulated for each delay quintile

\begin{tabular}{|l|r|r|r|r|r|}
\hline & \multicolumn{5}{|c|}{ Delay Quintile } \\
\hline Variable & 1 & 2 & 3 & 4 & 5 \\
\hline Delay (\%) & 0.10 & 0.17 & 0.22 & 0.26 & 0.34 \\
\hline Circuity (\%) & 0.28 & 0.31 & 0.30 & 0.32 & 0.34 \\
\hline Entropy & 0.57 & 0.61 & 0.45 & 0.31 & 0.37 \\
\hline $\begin{array}{l}\text { VKT per capita } \\
(\mathrm{km})\end{array}$ & 38.58 & 37.59 & 36.04 & 34.32 & 35.01 \\
\hline $\begin{array}{l}\text { Roadways per capita } \\
(\mathrm{km})\end{array}$ & 5.73 & 5.97 & 5.44 & 5.79 & 4.98 \\
\hline $\begin{array}{l}\text { Journey to work time } \\
(\mathrm{min})\end{array}$ & 23.79 & 24.42 & 26.63 & 26.61 & 28.87 \\
\hline $\begin{array}{l}\text { MSA population } \\
\text { (millions) }\end{array}$ & 1.48 & 1.53 & 3.54 & 4.91 & 6.22 \\
\hline
\end{tabular}




\section{Congestion $\left(\tau_{t t i}\right)$}

Congestion is a measure of network utilization. It depends on demand and supply. However, it depends on not just on the absolute quantity of these variables, but also on how they are organized.

The core hypotheses are:

- An increase in the demand, measured as population (density) of the area, increases congestion, ceterisparibus.

- An increase in the supply of the system decreases congestion.

An increase in roadway kilometers in the area decreases congestion

An decrease in the treeness of the network decreases congestion. Treeness measures network organizational inefficiency.

An increase in the entropy of the network decreases congestion. Entropy is a measure of the heterogeneity in the network and captures the presence of higher functional classification roads in the system.

Table 3 uses the basic model of congestion to understand the relationship between network structure and congestion. The congestion in the urban area is given by the Travel Time Index (TTI), provided as part of the the Urban Mobility Report. The TTI is a ratio that measures the travel time in the peak period to the travel time under free-flow conditions (Schrank and Lomax 2009). A higher value of TTI indicates higher congestion.

The TTI in an urban area is used as a dependent variable in this model. Alternate formulations of the proposed models - using different combinations of the independent variables and different functional forms (e.g., linear, translog, etc.), were tested. The results of the log-log regression model (robust standard errors) are presented in Table 3.

The results presented in Table 3 identify the factors that influence congestion. All independent variables perform as expected. Our hypothesis argued for an increase in congestion due to an increase in demand. This is confirmed by the positive and significant coefficient for the population and population density variables. The supply variable, namely, the total length of roads, has a negative influence on congestion as hypothesized.

The entropy of the roadway network had a significant negative influence on congestion. This corroborates our hypothesis that a more heterogeneous network leads to lesser congestion. The treeness in the roadway network has a positive and significant influence on congestion. This is in line with our hypothesis that a minimally connected tree network leads to congestion on the existing system due to the lack of travel options. The results show that the network structure affects system performance, after accounting for other non-network control variables.

The models presented here use the network measures as independent variables in explaining congestion and system usage in a region. Both the models presented here are log-log models and the elasticity estimates can therefore directly obtained from the respective model coefficients. Looking at the model results, we can say that a 1 percent increase in treeness of the roadway network increases the congestion in the area by 0.07 percent.

\section{System usage - DVKT per capita $\left(U_{m}\right)$}

System usage $\left(U_{m}\right)$ is measured as daily vehicle kilometers traveled (DVKT) per capita on all the roadways in the urban area, obtained from HPMS data. The actual measures of network structure $\left(N_{S}\right)$ that 
influence the network quantity and quality are identified based on an understanding of the transportation system.

Network structure $\left(N_{m}\right)$ comprises several measures, and how they operate is not always straightforward. On the one hand, improving efficiency lowers the network distance or travel time required to reach destinations, and thus, for a given set of activities, reduces the required travel. On the other hand, reducing distance required for a given set of destinations may lead to induced demand, whereby lowering net cost of traveling on the network point to point leads people to make longer trips. It is anticipated that the efficiency for given trips will outweigh induced demand effects, but this is, in the end, an empirical question that cannot be resolved by theory.

The core hypotheses are itemized below.

- An increase in the percentage of freeways, measuring the speed on the network, will increase DVKT. Higher hierarchy links such as freeways are usually faster and are more suited for longer travel.

- An increase in the completeness of the network, measuring the efficiency in network connectivity between origins and destinations, decreases DVKT per capita.

- An increase in the circuity, measuring the network inefficiency at the OD trip level, will increase the DVKT per capita. Higher circuity indicates greater inefficiency between OD pairs as travelers need to use more circuitous route (greater network distance) to reach their destinations.

- An increase in the street density, representing the quantity or supply of the network, increases the DVKT per capita. This is in line with the induced demand hypothesis, where increases to roadway capacity or supply encourage people to drive more by reducing the cost of travel.

- An increase in accessibility to population reduces the need for longer distance travel, and thus decreases the system usage.

- Price $\left(\tau_{t t i}\right)$ of highway travel measured in congestion, using the Travel Time Index (ratio of congested to freeflow time) decreases DVKT per capita. We posit that travelers respond to an increase in price by reducing travel.

Table 4 presents two models of system usage using DVKT per capita as the dependent variable. As with the congestion model, various formulations of the model using different functional forms and different combinations of the independent variables were tested. For brevity, only two selected models are presented and elaborated upon here.

Both models explain DVKT per capita as a function of network and non-network variables. The difference between the two models lies in the non-network variables considered in the analysis. The first model, Model A, uses the predicted congestion from Table 3 as a variable in explaining system usage. Model B, on the other hand, replaces the congestion variable with the measure of accessibility. Both models use the same measures of network structure: street density, percentage of freeways, completeness and circuity. Other common explanatory variables between the two models include auto mode share and median household income in the area.

The use of predicted congestion in Model A, rather than actual congestion, is to account for the causality between network congestion and usage. The congestion in a region is a function of the system usage, and the system usage in turn is a function of the congestion. The existence of this causality was confirmed by separate analyses including actual congestion measures (TTI, weighted average speed) in the models of system usage.

The results from both the models of system usage, presented in Table 4, confirm the influence of network structure on system usage. Focusing on the measures of network structure, the street density 
has a significant positive influence on DVKT per capita in both models, confirming our hypothesis that a larger supply of the street network of an urban area encourages more travel. The percentage of freeways in the area is also significant and positive, as hypothesized. Higher hierarchy links such as freeways are faster and are suited for longer travel. An increase in the percentage of freeways in the area increases DVKT per capita. The coefficient for the completeness in the network, measuring the efficiency in network connectivity, is negative in both models but is significant only in Model A. An efficiently connected network reduces the DVKT by providing good connectivity between the origins and destinations in the region and bringing them closer, reducing the need for travel. The average trip circuity is significant and negative in Model B and while positive in Model A. As noted previously, the effects of network structure are complex, and proper interpretation, given the sensitivity of this variable to model specification, require further investigation. The results from both models corroborate that aspects of network structure influence the performance of the transportation system.

The socio-demographic measures of auto mode share, median household income, and accessibility in the urban area show the expected influence on DVKT per capita. On a side note, Model B, with the inclusion of accessibility, shows a much better model fit, confirmed by the higher $R^{2}$. The better model fit with the inclusion of accessibility shows that the estimated accessibility is a better combination of congestion and density than using these variables independently and linearly in the regression models. As El-Geneidy and Levinson (2006) point out, using measures of mobility or congestion to understand the land use and transportation interaction is insufficient. Cities with the highest congestion might not be desirable places to live from a mobility (congestion) viewpoint, but are still attractive to residents because of the opportunities that they provide.

Table 3: Congestion (TTI)

\begin{tabular}{|l|c|c|c|}
\hline \multicolumn{4}{|c|}{ Dependent variable $(\ln )$ : Congestion (TTI) } \\
\hline Independent variables $(\ln )$ & Coef. & $\mathrm{t}$ & Sig. \\
\hline Population, $p$ & 0.09 & 5.01 & ${ }^{* * *}$ \\
\hline Population density, $\rho_{p m}$ & 0.04 & 1.88 & ${ }^{*}$ \\
\hline Total length of roads, $L_{r m}$ & -0.05 & -2.66 & ${ }^{* *}$ \\
\hline Entropy, $H(X)$ & -0.09 & -4.36 & ${ }^{* * *}$ \\
\hline Network treeness, $\phi_{\text {tree }}$ & 0.07 & 4.05 & ${ }^{* * *}$ \\
\hline Constant & -0.89 & -5.46 & ${ }^{* * *}$ \\
\hline Number of observations & 50 & & \\
\hline Adj. R-squared & 0.7380 & & \\
\hline Natural log of all variables considered in the analysis & & \\
\hline${ }^{*} \mathrm{p}<0.10,{ }^{* *} \mathrm{p}<0.05,{ }^{* *} \mathrm{p}<0.01$ & \multicolumn{3}{l}{} \\
\hline
\end{tabular}

\section{Conclusions}

This research aimed to develop quantitative measures that capture various aspects of network structure, using aggregate level travel data from fifty metropolitan areas across the US. The influence of these measures on system performance was then tested using two linear regression models. The first model analyzed the relationship between congestion and the estimated network measures. The second model analyzed relationship between the DVKT per capita and the network measures.

The results from both the models confirm that the quantitative measures of network structure affect the system performance, after controlling for independent variables that are non-network based. However, the influence of network design varies based on the aspect of travel that is being measured. 
Table 4: System Usage (DVKT) per capita

\begin{tabular}{|c|c|c|c|c|c|c|}
\hline \multicolumn{7}{|c|}{ Dependent variable (ln): System usage (DVKT) per capita } \\
\hline & \multicolumn{3}{|c|}{ Model A } & \multicolumn{3}{|c|}{ Model B } \\
\hline Independent variables $(\ln )$ & Coef. & $\mathrm{t}$ & Sig. & Coef. & & Sig. \\
\hline Predicted congestion, $\tau_{t t i}$ & -0.34 & -1.86 & * & NA & NA & NA \\
\hline Accessibility to population, $\mathrm{O}_{30}$ & NA & NA & NA & -0.52 & -8.34 & *** \\
\hline Percentage of freeways, $\% F$ & 0.26 & 2.38 & ** & 0.35 & 7.36 & *** \\
\hline Network completeness, $\rho_{e}$ & -0.21 & -3.89 & *** & -0.05 & -1.22 & \\
\hline Average circuity, $C$ & 0.82 & 1.84 & * & -0.65 & -1.94 & * \\
\hline Street density, $\rho_{l m}$ & 0.36 & 2.58 & ** & 0.56 & 9.63 & *** \\
\hline Median household income & 0.49 & 1.76 & $*$ & 0.29 & 2.32 & ** \\
\hline Auto mode share & 1.43 & 3.73 & *** & 0.84 & 3.21 & *** \\
\hline Constant & -5.38 & -1.48 & & 6.28 & 3.83 & *** \\
\hline Number of observations & \multicolumn{3}{|c|}{48} & \multicolumn{3}{|c|}{48} \\
\hline Adj. R-squared & \multicolumn{3}{|c|}{0.5627} & \multicolumn{3}{|c|}{0.8285} \\
\hline \multicolumn{7}{|c|}{ Natural log of all variables considered in the analysis } \\
\hline${ }^{*} \mathrm{p}<0.10,{ }^{* *} \mathrm{p}<0.05,{ }^{* * *} \mathrm{p}<0.01$ & & & & & & \\
\hline
\end{tabular}

The model explaining congestion shows the influence of network treeness while the model explaining DVKT per capita shows the influence of accessibility, street density, completeness and the percentage of freeways in the urban area. While the regression models presented in this paper allow us to quantify the magnitude of influence, the more important question is why is this understanding essential and how can the results be applied?

A report by the United Nations indicates that more than half ( 54 percent) of the world population currently lives in urban areas (United Nations Population Division 2014). The urban area population is expected to increase to 66 percent by 2050 , adding another 2.5 billion people. Consistent with the trends seen in the recent decade, 90 percent of this increase is expected to be in developing countries of Asia and Africa. This exponential increase in urban population will of course create a huge pressure on the existing infrastructure. While the recent economic downturn has dampened infrastructure spending in many countries, including the US and Europe, the emerging markets in Asia and Africa still continue to invest very heavily in their infrastructure to meet the growing demand and to sustain economic growth (Aldred 2012; Urban Land Institute and Ernst \& Young 2013). Infrastructure spending worldwide is expected to grow to more than $\$ 9$ trillion by 2025 with emerging markets in Asia-Pacific expected to represent 60 percent of this growth (Pricewaterhouse Coopers LLP 2014).

The rapid pace of these investments combined with the magnitude of spending makes it important to understand the impact of network design. Another reason to consider it is the irreversible nature of transportation network decisions. Transportation is among the longest lasting artifacts of our civilization. If we look back, we see that we still use paths first trod hundreds and thousands of years ago. Further, the transportation sector is the biggest consumer of energy and one of the biggest sources of greenhouse gas (GHG) emissions. According to the US Environmental Protection Agency (EPA), transportation accounts for about 28 percent of GHG emissions, and GHG emissions have increased by about 18 percent since 1990 (United States Environmental Protection Agency 2014).

Considering the background presented above, it is absolutely essential that we consider network architecture in the design of new transportation facilities. We are at a stage worldwide where the transportation systems in the developed world are in mature stage while the developing countries are just getting started with designing new infrastructure. This provides us a valuable opportunity where we 
can apply the lessons learned from the mature transportation systems to help design efficient and sustainable facilities.

So how can the results from this paper be applied? The models presented here quantify the impacts of the various network attributes on travel. For example, consider a scenario with two areas with the same urban form in terms of population and employment density. We argue that, considering everything else to be the same, a one percent greater network connectivity (completeness), is associated with 0.21 percent less travel. The argument isn't that we build more highways to reduce travel. Rather, it is that we consider network connectivity along with other measures of urban form while designing new infrastructure. There are other variables that affect travel in a region, and this model is a simple representation of travel. But the results show that different network designs are associated with different levels of travel. A combination of network design measures can thus be used to bring about desired changes in travel patterns and result in better system performance.

\subsection{Limitations}

As with any study, this analysis has its limitations and more research is needed to enhance the current models. The models developed here focus primarily on quantifying street networks but travel in an area is affected by other transportation modes. For example, public transportation is a very critical travel mode at least in large metropolitan areas such as New York and Washington, D.C. Further, in the last decade, we have seen increasing levels of non-motorized travel in the US (Pucher et al. 2011).

Derrible and Kennedy (2009)'s research on transit network design shows that key components of network design have significant impact on ridership and transit system performance. Similarly, Marshall and Garrick (2010) showed that fundamental characteristics of street network design such as connectivity, density, and patterns have an extremely important effect on encouraging non-motorized travel. As part of this analysis on metropolitan areas, it is important to quantify the structure of transit and other non-motorized networks, and to see how this affects the performance of the transportation system such as modal share.

The analysis presented in this paper is a cross-sectional comparison across regions for one specific year. While this allows us to establish correlation between the variables, it does not allow us to establish causality. Further research, with time series data for multiple regions, is hence required to test for causality. Also, as Marshall (2005) and Anas et al. (1998) point out, the current spatial structure of cities is based on the changes made to the transportation system in prior years. A good understanding of the influence of network structure on system performance needs to consider the temporal aspect. Another reason to consider time series data is the shift in the policy framework. In the last decade, the focus of transportation policies, especially in developed countries, has shifted from building new infrastructure to maintaining/fixing the existing infrastructure and moving to a more sustainable transportation system.

As Akerman et al. (2000) discuss, transportation policy needs to consider the consider the economic, environmental, and social aspects and needs to be more sustainable especially when high quality, well-connected networks already exist. The Transportation White Paper, adopted by the European Commission in 2001, argues for a shift from just building new infrastructure to establishing a balance between the different transportation modes, integrating the transportation system, and ensuring sustainable development (European Commission and others 2001). The use of time series data will allow us to better capture such shifts and the associated changes in travel patterns.

Another limitation to consider is that the models were developed using data from the metropolitan areas in the US. As expected, the transportation systems in the US are very different compared to the systems in Asia and Africa. A good extension of this study would be to obtain travel and network data from other countries and analyze the relation between network structure and travel. Such an analysis, 
while difficult in terms of data availability, would be a very useful enhancement of the current models and would also allow us to identify location-specific factors.

This analysis is part of a comprehensive effort to understand the influence of network structure on travel. It is hoped that the macro-level analysis conducted in this paper across metropolitan areas will provide an understanding of the relationship between network structure and travel that can be used to develop network design guidelines that address current transportation problems.

\section{Acknowledgements}

The author would like to thank Dr. David Levinson for the research impetus and invaluable guidance. The author acknowledges Paul Anderson, École Polytechnique Fédérale de Lausanne, for his invaluable assistance with the data collection efforts. Dr. Ahmed El-Geneidy, McGill University, provided the initial datasets for the study. 


\section{References}

Akerman, J., D. Banister, K. Dreborg, P. Nijkamp, R. Schleicher-Tappeser, D. Stead, and P. Steen. 2000. European transport policy and sustainable mobility. Routledge.

Aldred, D. 2012. Urbanization: A Major Driver of Infrastructure Spending. Citi Perspectives, 6(Q1/Q2).

Anas, A., R. Arnott, and K. Small. 1998. Urban spatial structure. Journal of Economic Literature, 36(3):1426-1464.

Barabasi, A. and E. Bonabeau. 2003. Scale-free networks. Scientific American, 288(5):50-59.

Bento, A., M. Cropper, A. Mobarak, and K. Vinha. 2003. The impact of urban spatial structure on travel demand in the United States, Volume 1. The World Bank, Washington D.C. Policy Research Working Paper Series: WPS 3007, URL http://go.worldbank.org/3GPQP2UWD0.

Borchert, J. 1961. The Twin Cities Urbanized Area: Past, Present, Future. Geographical Review, 51(1):47-70.

Crane, R. 2000. The Influence of Urban Form on Travel: An Interpretive Review. Journal of Planning Literature, 15(1):3-23.

Derrible, S. and C. Kennedy. 2009. A network analysis of subway systems in the world using updated graph theory. Transportation Research Record, 2112:17-25.

Derrible, S. and C. Kennedy. 2010. Characterizing metro networks: state, form, and structure. Transportation, 37(2):275-297.

El-Geneidy, A. M. and D. M. Levinson. 2006. Access to Destinations: Development of Accessibility Measures. St. Paul, MN: Minnesota Department of Transportation, Research Services Section; Available through the National Technical Information Service, Springfield, VA. Report 1 in the series: Access to Destinations Study, URL http://www.lrrb.org/PDF/200616.pdf.

Erath, A., M. Löchl, and K. Axhausen. 2009. Graph-theoretical analysis of the Swiss road and railway networks over time. Networks and Spatial Economics, 9(3):379-400.

European Commission and others. 2001. White paper: European transport policy for 2010: time to decide. Office for Official Publication of the European Communities, L-2985 Luxembourg.

Ewing, R. and R. Cervero. 2010. Travel and the built environment: A meta-analysis. Journal of the American Planning Association, 76(3):265-294.

Federal Highway Administration. 2000a. FHWA Functional Classification Guidelines. http://www.fhwa.dot.gov/planning/fcsec2_1.htm. Accessed August 2011.

Federal Highway Administration. 2000b. Highway Statistics Report. http://www.fhwa.dot.gov/policyinformation/hpms.cfm.

Federal Highway Administration. 2001. National Household Travel Survey. http://nhts.ornl.gov.

Garrison, W. L. and D. F. Marble. 1961. The structure of transportation networks. Evanston, Ill.: Transportation Center at Northwestern University. Draft of a report submitted October 31, 1961 to U.S. Army Transportation Research Command, Fort Eustis, Virginia, by the Transportation Center at Northwestern University under contract DA-44-177-TC-685, Transportation geography study.

Giuliano, G. and D. Narayan. 2003. Another look at travel patterns and urban form: The US and Great Britain. Urban Studies, 40(11):2295.

Gomez-Ibanez, J. 1991. A global view of automobile dependence. Journal of the American Planning Association, 57(3):376-379.

Gordon, P. and H. Richardson. 1989. Gasoline consumption and cities: A reply. Journal of the American Planning Association, 55(3):342-346.

$\mathrm{Gu}, \mathrm{X}$., D. Li, and L. Qin. 2012. Spatial structural characteristics of chinese railway passenger network based on complex network theory. EITA, 20(1):1.

Haggett, P. and R. Chorley. 1969. Network analysis in geography. London, U.K.: Edward Arnold. 
Hansen, W. 1959. How Accessibility Shapes Land Use. Journal of the American Planning Association, 25(2):73-76.

Horton, F. and D. Reynolds. 1971. Effects of urban spatial structure on individual behavior. Economic Geography, 47(1):36-48.

Jenelius, E. 2009. Network structure and travel patterns: Explaining the geographical disparities of road network vulnerability. Journal of Transport Geography, 17(3):234-244. ISSN 0966-6923.

Jiang, B. and C. Claramunt. 2004. Topological analysis of urban street networks. Environment and Planning B, 31(1):151-162.

Kansky, K. 1963. Structure of Transportation Networks: Relationships Between Network Geometry and Regional Characteristics. Ph.D. thesis, University of Chicago. Research Paper No. 84.

Kim, K., L. Benguigui, and M. Marinov. 2003. The fractal structure of Seoul's public transportation system. Cities, 20(1):31-39.

Kissling, C. 1969. Linkage Importance in a Regional Highway Network. The Canadian Geographer, 13(2):113-127.

Krizek, K. 2003. Operationalizing Neighborhood Accessibility for Land Use-Travel Behavior Research and Regional Modeling. Journal of Planning Education and Research, 22(3):270-287.

Levine, J., J. Grengs, and Q. Shen. 2009. Metropolitan accessibility and transpor sustainability: Comparitive indicators for policy reform. In Fifth Urban Research Symposium 2009: Cities and Climate Change: Responding to an Urgent Agenda. Marseille, France. URL http://siteresources.worldbank.org/INTURBANDEVELOPMENT/Resources/ 336387-1256566800920/6505269-1268260567624/Levine.pdf.

Levinson, D. 2012. Network structure and city size. PloS ONE, 7(1):e29721.

Levinson, D. and A. El-Geneidy. 2009. The minimum circuity frontier and the journey to work. Regional Science and Urban Economics, 39(6):732-738.

$\mathrm{Lu}, \mathrm{Y}$. and J. Tang. 2004. Fractal dimension of a transportation network and its relationship with urban growth: a study of the Dallas-Fort Worth area. Environment and Planning B, 31:895-912.

Marshall, S. 2005. Streets \& Patterns. New York: Spon Press.

Marshall, W. E. and N. W. Garrick. 2010. Effect of street network design on walking and biking. Transportation Research Record: Journal of the Transportation Research Board, 2198(1):103-115.

Newman, P. and J. Kenworthy. 1989. Gasoline consumption and cities: A comparison of U.S cities with a global survey. Journal of the American Planning Association, 55(1):24-37.

Parthasarathi, P., D. Levinson, and H. Hochmair. 2013. Network structure and travel time perception. Plos One, 8(10):e77718.

Patuelli, R., A. Reggiani, P. Nijkamp, and F. Bade. 2010. The evolution of the commuting network in Germany: Spatial and connectivity patterns. Journal of Transport and Land Use, 2(3/4):5-37.

Pricewaterhouse Coopers LLP. 2014. Capital project and infrastructure spending: Outlook to 2025. http://www.pwc.com/gx/en/capital-projects-infrastructure/publications/cpioutlook/about.jhtml. Research by Oxford Economics.

Pucher, J., R. Buehler, and M. Seinen. 2011. Bicycling renaissance in North America? An update and re-appraisal of cycling trends and policies. Transportation research part A: policy and practice, 45(6):451-475.

Salingaros, N. A. 1998. Theory of the urban web. Journal of Urban Design, 3(1):53-71.

Salingaros, N. A. 2000. Complexity and urban coherence. Journal of Urban Design, 5(3):291-316.

Schrank, D. and T. Lomax. 2009. 2009 Urban Mobility Report. Texas Transportation Institute, The Texas A\&M University System.

Schwanen, T., F. Dieleman, and M. Dijst. 2001. Travel behaviour in Dutch monocentric and policentric urban systems. Journal of Transport Geography, 9(3):173-186. 
Stead, D. 2001. Relationships between land use, socioeconomic factors, and travel patterns in Britain. Environment and Planning B, 28(4):499-528.

United Nations Population Division. 2014. World Urbanization Prospects: 2014 Revision. United Nations Publications.

United States Environmental Protection Agency. 2014. Sources of Greenhouse Gas Emissions. http://www.epa.gov/climatechange/ghgemissions/sources/transportation.html. Accessed July 2014.

Urban Land Institute and Ernst \& Young. 2013. Infrastructure 2013: Global Priorities, Global Insights. Washington, D.C. Accessed July 2014.

U.S. Census Bureau. 2008. Topologically Integrated Geographic Encoding and Referencing system (TIGER) Overview. Accessed 2009 July 4, URL http://www.census.gov/geo/www/tiger/ overview.html.

van de Coevering, P. and T. Schwanen. 2006. Re-evaluating the impact of urban form on travel patternsin Europe and North-America. Transport policy, 13(3):229-239.

Veneri, P. 2010. Urban polycentricity and the costs of commuting: evidence from italian metropolitan areas. Growth and Change, 41(3):403-429.

Xie, F. and D. Levinson. 2007. Measuring the structure of road networks. Geographical Analysis, 39(3):336-356. ISSN 0016-7363.

Yan, X. and M. Wang. 2009. Topological Properties of Public Transport Networks: A Temporal Perspective. In ICCTP 2009 critical issues in transportation system planning, development, and management: proceedings of the Ninth International Conference of Chinese Transportation Professionals: August 5-9, Harbin, China. Reston, VA: American Society of Civil Engineers.

Yang, B., X. Luan, and Q. Li. 2009. An adaptive method for identifying the spatial patterns in road networks. Computers, Environment and Urban Systems, 34:40-48.

Zhang, Y., J. Bigham, Z. Li, D. Ragland, and X. Chen. 2012. Associations between road network connectivity and pedestrian-bicyclist accidents. In 91st Annual Meeting of the Transportation Research Board, Washington DC. Washington, D.C. 